\title{
Streptozotocin: Its Excretion and Metabolism in the Rat
}

\author{
E. H. Karunanayake*, D. J. Hearse**, and G. Mellows* \\ Department of Biochemistry, Imperial College of Science and Technology, South Kensington, and \\ The Myocardial Metabolism Research Laboratories, The Rayne Institute, St. Thomas' Hospital, London, UK
}

Summary. The excretion of radioisotope following the administration of three specifically ${ }^{14} \mathrm{C}$-labelled forms of streptozotocin was investigated in the rat using ureter and bile duct cannulation techniques. The urine collected during the first hour following the administration of the drug contained the highest proportion of injected radioactivity (approximately 34\% with (3'-methyl- $\left.{ }^{14} \mathrm{C}\right)$-streptozotocin and approximately $40 \%$ each with $\left(1-{ }^{14} \mathrm{C}\right)$-and $\left(2^{1}-{ }^{14} \mathrm{C}\right)$-streptozotocin. Over the entire experimental period (6 hours), approximately $70 \%$ of the injected radioactivity of $\left(1-{ }^{14} \mathrm{C}\right)$ - and $\left(2^{1}-{ }^{14} \mathrm{C}\right)$-streptozotocin appeared in the urine. With ( $3^{\prime}$-methyl- $\left.{ }^{14} \mathrm{C}\right)$-streptozotocin, only $53 \%$ of the injected radioactivity appeared in the urine over the same period. In contrast to the high urinary excretion, less than $3 \%$ of the injected radioactivity from all three radiolabelled streptozotocin samples appeared in the bile. The in vivo and in vitro metabolism of streptozotocin was also investigated. In addition to substantial amounts of unchanged drug, three radiolabelled metabolites (two major and one minor) were detected in the urine during the 6 hour collection period following the administration of $\left(1-{ }^{14} \mathrm{C}\right)$ - and $\left(2^{\prime}-{ }^{14} \mathrm{C}\right)$-streptozotocin. In contrast, only unchanged $\left(3^{\prime}\right.$-methyl $\left.-{ }^{14} \mathrm{C}\right)$-streptozotocin was detected in the urine collected over the same period following the administration of the methyl labelled drug. The two major metabolites were also produced when $\left(1-{ }^{14} \mathrm{C}\right)$ and $\left(2^{1}-{ }^{14} \mathrm{C}\right)$-streptozotocin were incubated with a rat liver supernatant fraction $(100,000 \times \mathrm{g})$. The liver was further demonstrated to be the major site of metabolism in isolated liver perfusion studies in which both $\left(1-{ }^{14} \mathrm{C}\right)$ - and $\left(2{ }^{1}-{ }^{14} \mathrm{C}\right)$-streptozotocin were quan-

\footnotetext{
* Present address: Department of Biochemistry,

Bandaranaike Memorial Ayurvedic Research Institute, Nawinna, Maharagama, Sri Lanka.

** to whom reprint requests should be sent
}

titatively converted to the two major metabolites. The two major metabolites of $\left(1-{ }^{14} \mathrm{C}\right)$-streptozotocin, whether produced in vivo or in vitro, were chromatographically homogeneous with the two major metabolites formed from $\left(2^{1}-{ }^{14} \mathrm{C}\right)$-streptozotocin. Nicotinamide pretreatment had no apparent effect on the urinary excretion of streptozotocin and its metabolites.

Key words: Radiolabelled streptozotocin, metabolism, diabetes, urinary and biliary excretion, liver perfusion, nicotinamide pretreatment.

As part of the study into the metabolism and mode of action of the diabetogenic drug streptozotocin, we have reported [1] the synthesis of three specifically ${ }^{14} \mathrm{C}$-labelled forms of streptozotocin and have studied the distribution of each form in the rat. After the injection of $\left(1-{ }^{14} \mathrm{C}\right)$-streptozotocin or $\left(2{ }^{\prime}-{ }^{14} \mathrm{C}\right)$-streptozotocin, approximately $64 \%$ and $74 \%$, respectively, of the administered radioactivity appeared in the urine within 6 hours of administration. In contrast, after the injection of $\left(3^{\prime}-\right.$-methyl- $\left.{ }^{14} \mathrm{C}\right)$-streptozotocin, only $37 \%$ of the injected radioactivity was excreted during the same period. In an attempt to explain this observation and also to elucidate the metabolic fate and mode of action of streptozotocin, additional studies have been carried out.

In order to completely characterize the routes of excretion of various fragments of the streptozotocin molecule, to evaluate the possible role of liver and bile in the elimination of the drug and also to overcome the difficulty of collecting serial urine and bile samples from a conscious rat over a 6 hour period, cannulation studies were carried out. These studies were followed 
by a series of in vivo and in vitro investigations in which the metabolism of streptozotocin was characterized.

\section{Materials and Methods}

Radiolabelled Streptozotocin. $\left(1-{ }^{14} \mathrm{C}\right)$-streptozotocin, $\left(2^{\prime}-{ }^{14} \mathrm{C}\right)$-streptozotocin and $\left(3^{\prime}\right.$-methyl $\left.-{ }^{14} \mathrm{C}\right)$-streptozotocin (specific activity $1-2.7 \mu \mathrm{Ci} / \mathrm{mg}$ ) were synthesized as previously reported [1].

Experimental Animals. Sprague-Dawley rats maintained on a standard diet were used in all studies. For excretion experiments and in vivo metabolism studies male rats of $200 \pm 10 \mathrm{~g}$ body wt. were used. In in vitro metabolism studies livers were obtained from male rats of $400 \pm 10 \mathrm{~g}$ body wt.

Anaesthetization Procedures. During the intravenous administration of the drug all rats were lightly anaesthetized with sodium pentobarbitone $(15 \mathrm{mg} / \mathrm{kg}$ body wt., intraperitoneally) and anaesthesia was maintained for the duration of the experiment by intravenous administration of additional pentobarbitone as required.

Route of Administration and Dosage. In all investigations streptozotocin ( $70 \mathrm{mg} / \mathrm{kg}$ body wt.) was administered intravenously (usually via a lateral tail vein) while the animal was under light diethyl ether anaesthesia. In cannulation experiments the drug was administered via a cannula implanted in the jugular vein. Due to the instability of streptozotocin in aqueous media, all solutions $(70 \mathrm{mg} / \mathrm{ml})$ were prepared in sodium citrate buffer $(0.01 \mathrm{M}, \mathrm{pH} 4.5)$ immediately before administration.

Collection of Urine from Conscious Animals. After the injection of the radiolabelled drug, rats were housed in individual metabolism chambers and the total urine produced over a 6 hour period was collected.

Cannulation of the Trachea and the Jugular Vein. After the rat was anaesthetized the trachea was exposed and cannulated towards the lungs (tracheal cannulation was routinely carried out to prevent the occurrence of respiratory difficulties resulting from the accumulation of mucus in the upper regions of the respiratory tract). The right jugular vein was then exposed, incised and cannulated towards the heart.

Cannulation of the Ureters and the Bile Duct. Following the cannulation of the trachea and jugular vein, the abdominal cavity was opened by mid-line incision. The ureters were exposed and cannulated towards the kidneys so that the cannulae entered the renal pelvis. The bile duct was located and was cannulated towards the liver.
Collection of Urine and Bile from Cannulated Animals. Urine and bile samples from cannulated animals were separately collected into citrate buffer after 10 , $20,30,40,50,60,90,120,180,240,300,360$ minutes.

Liver Perfusion Studies. The perfusion apparatus and surgical procedures were essentially those described by Hems et al [2]. All perfusions were carried out at $37^{\circ} \mathrm{C}$. The perfusion medium contained $\mathrm{NaHCO}_{3}$-based saline $(63 \mathrm{ml})$, fraction $\mathrm{V}$ bovine serum albumin $(7 \mathrm{ml}, 15 \% \mathrm{w} / \mathrm{v})$ and glucose $(0.14 \mathrm{ml}$, $2 \mathrm{mM}$ ). The first $20 \mathrm{ml}$ of the effluent perfusion medium from the liver was discarded and then recirculating perfusion was initiated. The flow rate of the perfusate was adjusted to approximately $17 \mathrm{ml} / \mathrm{mi}$ nute. Perfusion was maintained for 30 minutes prior to the addition of streptozotocin $(500 \mathrm{mg}$ in $3 \mathrm{ml}$ of citrate buffer) to the perfusate. The perfusion was then maintained for an additional 120 minutes, during which time several samples of perfusate could be obtained. During the experiment bile was collected via a cannula inserted in the duodenum.

Incubation Studies. Rat liver (10 g) was homogenized in phosphate buffer $(0.01 \mathrm{M}, \mathrm{pH} 7.4)$ in a Waring blender and was centrifuged at $100,000 \times \mathrm{g}$ for 1 hour at $4^{\circ} \mathrm{C}$. The supernatant $(40 \mathrm{ml})$ was incubated with streptozotocin $(50 \mathrm{mg})$ for 6 hours at $37^{\circ} \mathrm{C}$.

Deproteinization. Protein present in the liver perfusion medium or incubation mixture was removed by ultrafiltration using Diaflo membranes (PM 10, Amicon Corp., Lexington, Mass., USA) under a gas $\left(0_{2}\right.$ free $\mathrm{N}$ ) pressure of $20 \mathrm{psi}$ and the filtrate was then freeze-dried.

\section{Chromatography}

Thin-layer and Preparative Layer Chromatography. Thin-layer chromatography (t.l.c.) was performed on Polygram CEL $300 \mathrm{UV}_{254}$ and Polygram Sil/UV 254 (0.1 mm thickness) precoated chromatograph sheets (Camlab, Cambridge, UK). Preparative layer chromatography was performed on cellulose MN 300 $\mathrm{UV}_{254}$ (1 mm thickness, loading not more than $50 \mathrm{mg}$ samples $/ 20 \times 20 \mathrm{~cm}$ plate). The solvent systems used were: Solvent A, butan-1-ol-acetic acid-water (3:1:1, $\mathrm{v} / \mathrm{v}$ ); Solvent B, ethyl acetate-acetic acid-formic acidwater $(18: 2: 1: 4, \mathrm{v} / \mathrm{v})$; Solvent C, propan-1-ol-ethyl acetate-water- $25 \%$ aqueous ammonia $(5: 1: 3: 1, \mathrm{v} / \mathrm{v})$.

Paper Chromatography. Descending paper chromatography was performed on Whatman No. 1 paper in chromatography tanks which had been pre-equilibrated for 8 hours with the appropriate solvent. The solvent front was allowed to run off the paper. Nacetylglucosamine was used as the standard and $R_{\mathrm{Nag}}$ is defined as: 
Table 1. The urinary biliary excretion of the three radiolabelled forms of streptozotocin

\begin{tabular}{|c|c|c|c|c|c|c|}
\hline \multirow{2}{*}{$\begin{array}{l}\text { Collection period } \\
\text { after } \\
\text { administration } \\
(\mathrm{min})\end{array}$} & \multicolumn{4}{|c|}{ Percentage recovery of injected radioactivity } & \multicolumn{2}{|c|}{$\left(3^{\prime}-\mathrm{Me}-{ }^{14} \mathrm{C}\right)$-streptozotocin } \\
\hline & Urine & Bile & Urine & Bile & Urine & Bile \\
\hline $0-10$ & $4.1 \pm 0.6$ & $0.05 \pm 0.0$ & $2.8 \pm 0.3$ & $0.06 \pm 0.0$ & $8.7 \pm 1.3$ & $0.03 \pm 0.0$ \\
\hline $10-20$ & $10.9 \pm 0.7$ & $0.06 \pm 0.0$ & $12.6 \pm 1.1$ & $0.08 \pm 0.0$ & $13.2 \pm 0.9$ & $0.03 \pm 0.0$ \\
\hline $20-30$ & $6.9 \pm 0.3$ & $0.07 \pm 0.0$ & $8.3 \pm 0.6$ & $0.07 \pm 0.0$ & $7.7 \pm 0.5$ & $0.03 \pm 0.0$ \\
\hline $30-40$ & $4.6 \pm 0.3$ & $0.08 \pm 0.0$ & $5.5 \pm 0.3$ & $0.08 \pm 0.0$ & $5.3 \pm 0.5$ & $0.02 \pm 0.0$ \\
\hline $40-50$ & $3.4 \pm 0.5$ & $0.09 \pm 0.0$ & $4.6 \pm 0.4$ & $0.09 \pm 0.0$ & $3.3 \pm 0.3$ & $0.02 \pm 0.0$ \\
\hline $50-60$ & $4.5 \pm 0.9$ & $0.08 \pm 0.0$ & $3.8 \pm 0.4$ & $0.10 \pm 0.0$ & $2.5 \pm 0.2$ & $0.03 \pm 0.0$ \\
\hline $60-90$ & $7.3 \pm 0.8$ & $0.27 \pm 0.0$ & $8.5 \pm 0.5$ & $0.31 \pm 0.0$ & $3.8 \pm 0.3$ & $0.08 \pm 0.0$ \\
\hline $90-120$ & $6.4 \pm 0.7$ & $0.26 \pm 0.0$ & $5.8 \pm 0.5$ & $0.30 \pm 0.0$ & $2.4 \pm 0.2$ & $0.07 \pm 0.0$ \\
\hline $120-180$ & $7.8 \pm 0.8$ & $0.45 \pm 0.0$ & $9.8 \pm 0.7$ & $0.49 \pm 0.0$ & $2.4 \pm 0.2$ & $0.12 \pm 0.0$ \\
\hline $180-240$ & $5.3 \pm 0.6$ & $0.37 \pm 0.0$ & $5.9 \pm 0.5$ & $0.41 \pm 0.0$ & $1.5 \pm 0.1$ & $0.11 \pm 0.0$ \\
\hline $240-300$ & $3.9 \pm 0.5$ & $0.34 \pm 0.0$ & $4.1 \pm 0.3$ & $0.33 \pm 0.0$ & $1.1 \pm 0.1$ & $0.09 \pm 0.0$ \\
\hline $300-360$ & $3.0 \pm 0.4$ & $0.27 \pm 0.0$ & $3.0 \pm 0.2$ & $0.29 \pm 0.0$ & $0.9 \pm 0.1$ & $0.07 \pm 0.0$ \\
\hline Total & $68.1 \pm 1.4$ & $2.39 \pm 0.2$ & $74.7 \pm 1.2$ & $2.66 \pm 0.2$ & $52.8 \pm 1.6$ & $0.75 \pm 0.0$ \\
\hline
\end{tabular}

\section{$\mathrm{R}_{\mathrm{Nag}}=\frac{\text { Distance component travels from origin }}{\text { Distance } \mathrm{N} \text {-acetylglucosamine travels }}$ from origin}

The solvent systems used were: Solvent $D$, butan-1ol-ethanol-water $(52: 33: 15, \mathrm{v} / \mathrm{v})$ : Solvent E, propan-2-ol-water $(4: 1, \mathrm{v} / \mathrm{v})$. With all chromatograms radioactive spots were located with the aid of a spark chamber (Birchover Instruments, Bancroft, Hitchin, Herts, UK) and also with conventional autoradiographic techniques.

Nicotinamide Pretreatment Studies. In these studies nicotinamide (British Drug House, Poole, England) was administered ( $250 \mathrm{mg} / \mathrm{kg}$ body wt., dissolved in $1 \mathrm{ml}$ of physiological saline) intraperitoneally to conscious rats and via the jugular cannula to anaesthetized animals. Streptozotocin was administered 15 minutes after nicotinamide treatment. Urine samples were collected 6 hours following the injection of streptozotocin.

Radiochemical Techniques. The ${ }^{14} \mathrm{C}$ in urine and bile was determined in dioxane based scintillant [1], using a Beckman LS-200B liquid scintillation counter.

\section{Results}

\section{Excretion Studies}

In these studies streptozotocin $(70 \mathrm{mg} / \mathrm{kg}$ ) was administered intravenously to male rats in which cannulae had been implanted in the ureters and bile ducts. Urine and bile samples were collected for a total period of 6 hours, and samples were assayed for radioactivity. Six rats were used for each labelled form of the drug and the results are shown in Table 1 . These results, while in general agreement with the excretion patterns observed in experiments with conscious animals [3] reveal several additional interesting points. Firstly, the urine collected during the first 1 hour collection period following the administration of the drug contained the highest proportion of the injected radioactivity (34\% with $33^{\prime}$-methyl- ${ }^{14} \mathrm{C}$ )-streptozotocin, $40 \%$ each with $\left(1-{ }^{14} \mathrm{C}\right)$-streptozotocin and $\left(2^{\prime}-{ }^{14} \mathrm{C}\right)$-streptozotocin). This large proportion suggested that either unchanged streptozotocin or its metabolites were rapidly eliminated from the body. During the first hour, the peak urinary excretion for each of the three labelled forms of the drug occurred during the 10-20 minute period after administration.

On a time related basis, the peak of renal excretion coincided with the first peak of renal radioactivity observed in tissue distribution studies and also with the maximum renal uptake and accumulation of radioactivity revealed in autoradiographic experiments $[1,4]$. Over the entire experimental period, approximately $70 \%$ of the injected radioactivity for both $\left(1-{ }^{14} \mathrm{C}\right)$-streptozotocin and $\left(2^{\prime}-{ }^{14} \mathrm{C}\right)$-streptozotocin appeared in the urine. In contrast, with $\left(3^{1}\right.$-methyl $\left.-{ }^{14} \mathrm{C}\right)$-streptozotocin only $53 \%$ of the injected radioactivity was excreted in the urine during the same period. This observation suggests that, in the course of the metabolism of streptozotocin, the $\left(3^{\prime}\right.$-methyl) moiety is cleaved from the rest of the molecule and is retained in the body for a longer period of time. In contrast to the high urinary excretion of injected radioactivity, only a small proportion (less than $3 \%$ ) of the injected radioactivity appeared in the bile following the administration of all three 
radiolabelled forms of streptozotocin. In view of the factors which are known to limit biliary excretion [5, 6], the low level of radioactivity found in the bile is perhaps not surprising. Furthermore, this observation would suggest that the metabolites of streptozotocin have a relatively low molecular weight (i.e. less than $300)$.

\section{Metabolism Studies}

In Vivo Experiments. In an attempt to follow the metabolism of streptozotocin, urine samples were collected over a 6 hour period following the injection of either $\left(1-{ }^{14} \mathrm{C}\right)$-streptozotocin or $\left(2{ }^{1}-{ }^{14} \mathrm{C}\right)$-streptozotocin $(70 \mathrm{mg} / \mathrm{kg})$, from conscious rats housed in metabolism chambers. Urine and bile samples were also collected $(30,60,90,120,180,240,300$ and 360 minutes after injection) from anaesthetized rats with cannulated ureters and bile ducts. The urine and bile samples were subjected to t.l.c. on cellulose and the radioactive components were located by autoradiographic and spark chamber techniques. With $\left(1-{ }^{14} \mathrm{C}\right)$ streptozotocin, in addition to substantial amounts of unchanged streptozotocin, two major metabolites ( $\mathrm{M}_{1}$ and $\left.\mathrm{M}_{2}\right)$ and one minor metabolite $\left(\mathrm{M}_{5}\right)$ were detected in the urine. The chromatographic mobilities of $\mathrm{M}_{1}$, $\mathrm{M}_{2}$ and $\mathrm{M}_{5}$ in Solvent A were $0.55,0.51$ and 0.43 and in Solvent B were $0.33,0.26$ and 0.10 respectively. The results indicated that there was no difference in the metabolism of the drug between conscious animals and those maintained under anaesthesia for 6 hours. Similarly, the administration of $\left(2{ }^{\prime}-{ }^{14} \mathrm{C}\right)$-streptozotocin led to the appearance in the urine of unchanged streptozotocin plus two major metabolites $\left(\mathrm{M}_{3}\right.$ and $\left.\mathrm{M}_{4}\right)$ and one minor metabolite $\left(\mathrm{M}_{6}\right)$ which were chromatographically homogeneous with $\mathrm{M}_{1}, \mathrm{M}_{2}$ and $\mathrm{M}_{5}$ respectively. In contrast, only unchanged $\left(3^{3}\right.$-methyl $\left.{ }^{14} \mathrm{C}\right)$-streptozotocin could be detected in the 6 hour urine samples following its administration.

Effect of Nicotinamide Pretreatment on the Metabolism of Streptozotocin. In view of the reported [7] protective action of nicotinamide against streptozotocin-induced diabetes, the effect of nicotinamide on the metabolism of $\left(2^{\prime}{ }^{14} \mathrm{C}\right)$-streptozotocin was investigated in conscious and cannulated animals. Nicotinamide $250 \mathrm{mg} / \mathrm{kg}$ body wt. was administered 15 minutes prior to streptozotocin treatment (intraperitoneally to conscious animals and via the jugular vein to cannulated animals) and urine samples were collected over a 6 hour period. The t.l.c. pattern of urinary metabolites from nicotinamide- $\left(2^{1}-{ }^{14} \mathrm{C}\right)$ streptozotocin treated rats was identical with the t.l.c. pattern of urinary metabolites from rats treated with $\left(2{ }^{\prime}-{ }^{14} \mathrm{C}\right)$-streptozotocin only. In addition to the radioactive streptozotocin metabolites, t.l.c. analysis also revealed the presence of $\mathrm{N}$-methyl-nicotinamide, However, when the urine from nicotinamide-( 3 'methyl- ${ }^{14} \mathrm{C}$ )-streptozotocin treated animals was examined, the only radioactive component present was unchanged streptozotocin. No radioactivity could be detected in N-methyl-nicotinamide, which was also present. The latter observation would suggest that under the conditions of this study the $\mathrm{N}$-methyl group of $\mathrm{N}$-methyl-nicotinamide results from an endogenous source and not by simple transmethylation from streptozotocin. Similar conclusions were drawn from preliminary experiments with conscious animals.

In Vitro Studies. Initial attempts to isolate and identify the major metabolites were hampered by the fact that these substances were present in relatively small quantities, and exhibited very similar chromatographic properties. Furthermore, high levels of glucose (which had a similar chromatographic mobility to the major metabolites) appeared in the urine as a result of the induction of the diabetic state.

In an attempt to overcome these difficulties, and also to evaluate the possibility of preparing greater quantities of the metabolites, an in vitro study of the metabolism of $\left(1-{ }^{14} \mathrm{C}\right)$ - and $\left(2^{\prime}-{ }^{14} \mathrm{C}\right)$-streptozotocin was undertaken.

Incubation Experiments. T.l.c. analysis of the deproteinized and freeze-dried incubation mixture revealed a quantitative conversion of streptozotocin into several metabolites which were chromatographically homogeneous with those found in the urine samples from in vivo experiments, i.e. $M_{1}, M_{2}$ for $\left(1-{ }^{14} \mathrm{C}\right)$ streptozotocin and $\mathrm{M}_{3}, \mathrm{M}_{4}$ for $\left(2^{\prime}-{ }^{14} \mathrm{C}\right)$-streptozotocin respectively.

Liver Perfusion Studies. T.1.c. analysis of the deproteinized and freeze-dried perfusion medium revealed that during the course of perfusion there had been a quantitative conversion of $\left(1-{ }^{14} \mathrm{C}\right)$-streptozotocin or $\left(2^{\prime}-{ }^{14} \mathrm{C}\right)$-streptozotocin into the two major metabolites which were chromatographically identical with those produced in the in vivo studies. Analysis of bile collected during the perfusion period revealed only trace amounts of the two metabolites.

Chromatographic Studies of Isolated Metabolites. The two major metabolites $\mathrm{M}_{1}$ and $\mathrm{M}_{2}$ or $\mathrm{M}_{3}$ and $\mathrm{M}_{4}$, obtained from urine (6 hour sample), liver homogenate mixture and liver perfusion medium were separated by preparative layer chromatography on cellulose (Solvent A). The bands $R_{f} 0.55$ and $R_{f} 0.51$ were separately eluted and freeze-dried. These isolated metabolites were further chromatographed on Polygram Sil/ $\mathrm{UV}_{254}$ (Solvent $\mathrm{C}$ ) and paper chromatography (Solvent $D$ and $E$ ), and these studies confirmed that the chromatographic mobilities of $M_{1}\left(R_{f} 0.53\right.$ : $R_{\text {Nag }} 1.10$ and 0.85 respectively) and $M_{2}\left(R_{f} 0.42 ; R_{N a g}\right.$ 0.75 and 0.65 respectively) produced from $\left(1-{ }^{14} \mathrm{C}\right)-$ 
streptozotocin either by in vivo or in vitro experiments were identical to those $\left(\mathrm{M}_{3}\right.$ and $\mathrm{M}_{4}$ respectively) produced from $\left(2^{\prime}-{ }^{14} \mathrm{C}\right)$-streptozotocin. These observations suggest that the $\left(1{ }^{14} \mathrm{C}\right)$ and $\left(2^{\prime}-{ }^{14} \mathrm{C}\right)$ carbon atoms of streptozotocin are subjected to the same metabolic fate [8]. Furthermore, the results would suggest that the metabolism of streptozotocin must involve molecular cleavage between the 2'-carbon atom and the 3 -methyl group and the retention of the latter in the animal. The suscepibility of the C-2'-N-3' bond to scission at physiological $\mathrm{pH}$ would be expected since $\mathrm{N}$-methyl- $\mathrm{N}$-nitrosourea compounds of this type are unstable at slightly alkaline $\mathrm{pH}$, rapidly generating diazomethane through hydrolysis of this bond.

Such an event would be consistent with the observed incorporation of the $\left(1-{ }^{14} \mathrm{C}\right)$ and $\left(2{ }^{1}-{ }^{14} \mathrm{C}\right)$ carbon atoms into common metabolites and would also be compatible with known biological degradation patterns of $\mathrm{N}$-nitrosoureido compounds [9].

\section{Discussion}

These collective findings may suggest a possible mechanism for the diabetogenic action of the drug. The diabetogenic activity of streptozotocin has been attributed to its marked specificity for the B-cells of the Islets of Langerhans [10]. The biochemical basis of this damage has been suggested [11] to involve the streptozotocin-induced depletion of pancreatic $\mathrm{NAD}^{+}$concentration. This hypothesis is supported by the observation [7] that nicotinamide, when administered either 15 minutes before, or up to 2 hours after streptozotocin treatment, prevents the onset of diabetes. In contrast, nicotinic acid was ineffective as a protective agent. Several mechanisms $[7,12,11,13]$ have been suggested to explain the depletion of pancreatic $\mathrm{NAD}^{+}$concentration induced by streptozotocin.

We have investigated the possibility that streptozotocin may deplete cellular levels of $\mathrm{NAD}^{+}$by simple transmethylation to nicotinamide generating $\mathrm{N}$-methyl-nicotinamide, thereby removing nicotinamide available for $\mathrm{NAD}^{+}$biosynthesis. However, no radioactive $\mathrm{N}$-methyl-nicotinamide could be detected in the urine following the administration of $\left(3^{\prime}\right.$-methyl- $\left.{ }^{14} \mathrm{C}\right)$-streptozotocin to nicotinamide pretreated rats. This result is in agreement with in vitro studies reported by Dean \& Mathews [14]. It therefore seems unlikely that the protective action of nicotinamide can be explained in terms of its acting as an acceptor for the free methyl group of strcptozotocin. Conclusive verification of this point would, how- ever, require time studies using $\left(3^{1}\right.$-methyl $\left.{ }^{14} \mathrm{C}\right)$ streptozotocin of far higher specific activity than that used in these experiments. However, it is noteworthy that nicotinamide has been shown $[15,16]$ to be more efficient than nicotinic acid as a biosynthetic precursor of $\mathrm{NAD}^{+}$in the rat, and that the half-life of the former in both rat liver and blood is significantly longer than that of nicotinic acid. This observation may provide some explanation for the marked protective action of nicotinamide against streptozotocin-induced diabetes.

In conclusion, the results described in the present paper, confirm our previous reports $[1,8,17]$ that streptozotocin is subjected to rapid metabolic degradation by the rat. 'The results indicate that liver is the major site of metabolism of streptozotocin. The high urinary excretion and the lack of any significant biliary elimination would suggest that these metabolites are low molecular weight compounds.

Acknowledgements. E.H.K. was supported by a Colombo-Plan Fellowship. This work was supported in part by a grant from the British Heart Foundation. The assistance of Patricia Whitton with liver perfusion experiments is gratefully acknowledged.

\section{References}

1. Karunanayake, E. HI., Hearse, D. J., Mellows, G.: The synthesis of ${ }^{11} \mathrm{C}$-streptozotocin and its distribution and excretion in the rat. Biochem. J. 142, 673-683 (1974a)

2. Hems, R., Ross, B. D., Berry, M. N., Krebs, H. A.: Gluconeogenesis in the perfused rat liver. Biochem. J. 101, 284-292 (1966)

3. Karunanayake, E. H., Hearse, D. J., Mellows, G.: The distribution and excretion of $\left({ }^{14} \mathrm{C}\right)$-streptozotocin in the rat. Biochem. Soc. Trans. 2, 1006-1009 (1974b)

4. Karunanayake, E. H., Baker, J. R. J., Christian, R. A., Hearse, D. J., Mellows, G.: Autoradiographic study of the distribution and cellular uptake of $\left({ }^{14} \mathrm{C}\right)$-streptozotocin in the rat. Diabetologia 12, 1-6 (1976)

5. Milburn, P., Smith, R. L., Williams, R. T.: Biliary excretion of foreign compounds. Biochem. J. 105, 1275-1281 (1967a)

6. Milburn, P., Smith, R. L., Williams, R. T.: Biliary excretion of foreign compounds. Biochem. J. 105, 1283-1287 (1967b)

7. Dulin, W. E., Wyse, B. M.: Studies on the ability of compounds to block the diabetogenic action of streptozotocin. Diabetes 18 , $459-466$ (1969)

8. Karunanayake, E. H., Hearse, D. J., Mellows, G.: The metabolic fate and elimination of streptozotocin. Biochem. Soc. Trans. 3, 410-414 (1975a)

9. Lawley, P. D., Shah, S. A.: N-methyl-N-nitrosourea and Nmethyl- $\mathrm{N}^{1}$-nitro-N-nitrosoguanadine. Biochem. J. 128, 117132 (1972)

10. Rakienten, N., Rakienten, M. L., Nadkarni, M. V.: Studies on the diabetogenic action of streptozotocin. Cancer Chemother. Rep. 29, 91-98 (1963)

11. Schein, P. S., Cooney, D. A., McMenamin, M. G., Anderson, T.: Streptozotocin diabetes - further studies on the mechanisms 
of depression of nicotinamide adenine dinucleotide concentrations in mouse pancreatic islets and liver. Biochem. Pharmcol. 22, 2625-2631 (1973)

12. Schein, P. S., Loftus, S.: Streptozotocin: Depression of mouse liver pyridine nucleotides. Cancer Res. 28, 1501-1506 (1968)

13. Hinz, M., Katsilambros, N., Maier, V., Schatz, H., Pfeiffer, E. F.: Significance of streptozotocin induced nicotinamide adenine-dinucleotide (NAD) degradation in mouse pancreatic islets. FEBS Letters 30, 225-228 (1973)

14. Dean, P. M., Mathews, E. K.: The bioelectric properties of pancreatic islets cells: Effect of diabetogenic agents. Diabetologia 8, 173-178 (1972)

15. Petrack, B., Greengard, P., Kalinsky, H.: On the relative efficacy of nicotinamide and nicotinic acid as precursors of nicotinamide adenine dinucleotide. J. biol. Chem. 241, 2367-2372 (1966)

16. Collins, P. B., Chaykin, S.: The management of nicotinamide and nicotinic acid in the mouse. J. biol. Chem. 247, 778-783 (1972)

17. Karunanayake, E. H., Baker, J. R. J., Christian, R., Hearse, D. J., Mellows, G.: Microradioautographic study of the distribution and uptake of $\left({ }^{14} \mathrm{C}\right)$-streptozotocin in rat tissues. Biochem. Soc. Trans. 3, 414-417 (1975b)

Received: January 12, 1976, and in revised form: June 21, 1976

Dr. D. J. Hearse

The Rayne Institute

St. Thomas' Hospital

London SE1 7EH

England 